\title{
RESIDUATION THEORY AND MATRIX MULTIPLICATION ON ORTHOMODULAR LATTICES
}

\author{
by J. H. BEVIS and C. K. MARTIN
}

(Received 27 December, 1967)

In this paper we consider mappings induced by matrix multiplication which are defined on lattices of matrices whose coordinates come from a fixed orthomodular lattice $L$ (i.e. a lattice with an orthocomplementation denoted by ' in which $\left.a \leqq b \Rightarrow a \vee\left(a^{\prime} \wedge b\right)=b\right)$. $\mathscr{A}_{m n}$ will denote the set of all $m \times n$ matrices over $L$ with partial order and lattice operations defined coordinatewise. For conformal matrices $A$ and $B$ the $(i, j)$ th coordinate of the matrix product $A B$ is defined to be $(A B)_{i j}=\bigvee_{k}\left(A_{i k} \wedge B_{k j}\right)$. We assume familiarity with the notation and results of [1]. $\mathscr{A}_{m n}$ is an orthomodular lattice and the (lattice) centre of $\mathscr{A}_{m n}$ is defined as $\mathscr{C}\left(\mathscr{A}_{m n}\right)=\left\{A \in \mathscr{A}_{m n} \mid A \mathscr{C} B\right.$ for all $\left.B \in \mathscr{A}_{m n}\right\}$, where we say that $A$ commutes with $B$ and write $A \mathscr{C} B$ if $\left(A \vee B^{t}\right) \wedge B=A \wedge B$. In $\S 1$ it is shown that mappings from $\mathscr{A}_{m n}$ into $\mathscr{A}_{m r}$ characterized by right multiplication $X \rightarrow X P\left(P \in \mathscr{A}_{n r}\right)$ are residuated if and only if $P \in \mathscr{C}\left(\mathscr{A}_{n r}\right)$. (Similarly for left multiplication.) This result is used to show the existence of residuated pairs. Hence, in $\$ 2$ we are able to extend a result of Blyth [3] which relates invertible and cancellable matrices (see Theorem 3 and its corollaries). Finally, for right (left) multiplication mappings, characterizations are given in $\S 3$ for closure operators, quantifiers, range closed mappings, and Sasaki projections.

1. After Croisot [4] a monotone mapping $\phi: \mathscr{A} \rightarrow \mathscr{B}$ from a lattice $\mathscr{A}$ into a lattice $\mathscr{B}$ is residuated if there is a monotone mapping $\phi^{+}: \mathscr{B} \rightarrow \mathscr{A}$ called the residual mapping corresponding to $\phi$ such that $a \leqq a \phi \phi^{+}$for all $a$ in $\mathscr{A}$ and $b \phi^{+} \phi \leqq b$ for all $b$ in $\mathscr{B}$. One may show that $\phi$ and $\phi^{+}$determine each other uniquely.

THEOREM 1. Given $P \in \mathscr{A}_{n r}$, the mapping $\phi: \mathscr{A}_{m n} \rightarrow \mathscr{A}_{m r}$ defined by $A \phi=A P$ is residuated if and only if $P \in \mathscr{C}\left(\mathscr{A}_{n \mathrm{r}}\right)$. If $\phi$ is residuated, $B \phi^{+}=\left(B^{\prime} P^{t}\right)^{\prime}$, where $P^{t}$ is the transpose of $P$.

Proof. According to [4], a residuated mapping preserves joins. Hence, by Lemma 2 of [1], if $A \rightarrow A P$ is residuated, then $P \in \mathscr{C}\left(\mathscr{A}_{n r}\right)$. If $P \in \mathscr{C}\left(\mathscr{A}_{n r}\right)$, then

$$
\left[(A P)^{\prime} P^{t}\right]_{i j}=\bigvee_{k}\left[P_{j k} \wedge \bigwedge_{h}\left(A_{i h}^{\prime} \vee P_{h k}^{\prime}\right)\right]=\bigvee_{k}\left[P_{j k} \wedge A_{i j}^{\prime} \wedge \bigwedge_{h \neq j}\left(A_{i h}^{\prime} \vee P_{h k}^{\prime}\right)\right] \leqq A_{i j}^{\prime}
$$

Hence $A \leqq\left[(A P)^{\prime} P^{t}\right]^{\prime}$. Similarly $\left(B^{\prime} P^{t}\right)^{\prime} P \leqq B$.

For left multiplication we have the result:

THEOREM 1*. Given $P \in \mathscr{A}_{n r}$, the mapping $\phi: \mathscr{A}_{r m} \rightarrow \mathscr{A}_{n m}$ defined by $A \phi=P A$ is residuated if and only if $P \in \mathscr{C}\left(\mathscr{A}_{n r}\right)$. If $\phi$ is residuated, $B \phi^{+}=\left(P^{t} B^{\prime}\right)^{\prime}$.

Extending the definition of Birkhoff [2, XIII], for $P$ in $\mathscr{A}_{n r}$ and $B$ in $\mathscr{A}_{m r}$ ( $B$ in $\mathscr{A}_{n m}$ ), we define the right-residual $B: P$ (left-residual $B:: P$ ) of $B$ by $P$ as the largest $X$ in $\mathscr{A}_{m n}\left(\mathscr{A}_{r m}\right)$, if it exists, satisfying $X P \leqq B(P X \leqq B)$. Such a pair $P, B$ is said to be residuated on the right (left) if $B: P(B:: P)$ exists.

The first two lemmas are due to Croisot [4], and are used in the proof of Theorem 2. 
LEMMA 1. Let $\phi: \mathscr{A} \rightarrow \mathscr{B}$ be a residuated mapping, and let $\phi^{+}$be the corresponding residual mapping. For $b$ in $\mathscr{B}, b \phi^{+}$is the greatest element in the non-empty set $\{a \in \mathscr{A} \mid a \phi \leqq b\}$.

LEMMA 2. In order that the monotone mapping $\phi: \mathscr{A} \rightarrow \mathscr{B}$, where $\mathscr{A}$ and $\mathscr{B}$ are lattices, be residuated, it is necessary and sufficient that for every $b$ in $\mathscr{B}$ the set $\{a \in \mathscr{A} \mid a \phi \leqq b\}$ be nonempty and contain a greatest element.

THEOREM 2. For $P$ in $\mathscr{A}_{n r}$ the following conditions are equivalent:

(i) $P \in \mathscr{B}\left(\mathscr{A}_{n r}\right)$.

(ii) $B: P$ exists for all $B$ in $\mathscr{A}_{m r}$.

(iii) $B:: P$ exists for all $B$ in $\mathscr{A}_{n m}$.

Moreover, if $P \in \mathscr{C}\left(\mathscr{A}_{n r}\right)$ and $B \in \mathscr{A}_{m r}\left(B \in \mathscr{A}_{n m}\right)$, then $B: P=\left(B^{\prime} P^{t}\right)^{\prime}\left(B:: P=\left(P^{t} B^{\prime}\right)^{\prime}\right)$.

Proof. By Theorem 1 and Lemma 1, (i) implies (ii) and (iii). By Lemma 2 and Theorem 1, (ii) or (iii) implies (i).

2. Motivated by Molinaro [9], we define two types of equivalence relations. For $P$ in $\mathscr{C}\left(\mathscr{A}_{n r}\right)$ define the equivalence relation $\Psi_{P}$ on $\mathscr{A}_{m r}$ by $A \equiv B\left(\Psi_{P}\right)$ if $A: P=B: P$, and define the equivalence relation ${ }_{P} \Psi$ on $\mathscr{A}_{n m}$ by $A \equiv B\left({ }_{P} \Psi\right)$ if $A:: P=B:: P$. For $P \in \mathscr{A}_{r n}$ define the equivalence relation $\Theta_{P}$ on $\mathscr{A}_{n m}$ by $A \equiv B\left(\Theta_{P}\right)$ if $P A=P B$, and define the equivalence relation ${ }_{P} \Theta$ on $\mathscr{A}_{m r}$ by $A \equiv B\left({ }_{P} \Theta\right)$ if $A P=B P$.

Lemma 3. For $P$ in $\mathscr{C}\left(\mathscr{A}_{n r}\right)$, each class in $\mathscr{A}_{m r}\left(\mathscr{A}_{n m}\right)$ modulo $\Psi_{P}\left({ }_{P} \Psi\right)$ has a smallest element; the smallest element in the class containing $A$ is $(A: P) P(P(A:: P))$. For $P$ in $\mathscr{C}\left(\mathscr{A}_{\mathrm{rn}}\right)$, each class in $\mathscr{A}_{n m}\left(\mathscr{A}_{m r}\right)$ modulo $\Theta_{P}\left({ }_{P} \Theta\right)$ has a greatest element; the greatest element in the class containing $A$ is $P A:: P(A P: P)$.

Proof. Given $P \in \mathscr{C}\left(\mathscr{A}_{n r}\right)$ and $A \equiv B\left(\Psi_{P}\right)$ in $\mathscr{A}_{m r} ;$ then $(A: P) P=(B: P) P \leqq B$, i.e., $(A: P) P$ is well defined on the class containing $A$ and is a lower bound for the class. From $(A: P) P=(A: P) P$ we obtain $A: P \leqq(A: P) P: P$. Also, by the definition of right-residual, $[(A: P) P: P] P \leqq(A: P) P \leqq A$, which implies that $(A: P) P: P \leqq A: P$. Hence $(A: P) P \equiv A\left(\Psi_{P}\right)$. Given $P \in \mathscr{B}\left(\mathscr{A}_{n n}\right)$ and $A \equiv B\left(\Theta_{P}\right)$ in $\mathscr{A}_{n m}$, it follows that $P A:: P=P B:: P$. From $P A=P A$ we obtain $A \leqq P A:: P$, i.e., $P A:: P$ is well defined on the class containing $A$ in an upper bound for the class. Now $P A \leqq P(P A:: P)$ by monotonicity of multiplication and $P(P A:: P) \leqq P A$ by the definition of left-residual. Hence $P A:: P \equiv A\left(\Theta_{P}\right)$. The remaining two parts of the lemma follow in a similar manner.

We are now ready to extend a result of Blyth [3] for Boolean matrices, to matrices over orthomodular lattices.

Lemma 4. For $P$ in $\mathscr{A}_{r n}, A \equiv B\left(\Theta_{P}\right)$ in $\mathscr{A}_{n m} \Leftrightarrow A^{t} \equiv B^{t}\left({ }_{P t} \Theta\right)$ in $\mathscr{A}_{m n}$. For $P$ in $\mathscr{C}\left(\mathscr{A}_{n r}\right)$, $A \equiv B\left(\Psi_{P}\right)$ in $\mathscr{A}_{m r} \Leftrightarrow A^{t} \equiv B^{t}\left({ }_{p t} \Psi\right)$ in $\mathscr{A}_{r m}$.

Pronf. The first part is an immediate consequence of $(A P)^{t}=P^{t} A^{t}$. With $P$ in $\mathscr{C}\left(\mathscr{A}_{n r}\right)$, by Theorem 2 we obtain $A: P=\left(A^{\prime} P^{t}\right)^{\prime}=\left(P A^{\prime t}\right)^{\prime t}=\left(A^{t}:: P^{t}\right)^{t}$. Thus $A \equiv B\left(\Psi_{\mathrm{P}}\right)$ in $\mathscr{A}_{m r} \Leftrightarrow\left(A^{t}:: P^{t}\right)^{t}=\left(B^{t}:: P^{t}\right)^{t} \Leftrightarrow A^{t} \equiv B^{t}\left({ }_{P t} \Psi\right)$. 
Lemma 5. For $P$ in $\mathscr{C}\left(\mathscr{A}_{n r}\right), A \equiv B\left(\Theta_{p t}\right)$ in $\mathscr{A}_{n m} \Leftrightarrow A^{\prime} \equiv B^{\prime}\left({ }_{P} \Psi\right)$ in $\mathscr{A}_{n m}$, and $A \equiv B\left({ }_{p t} \Theta\right)$ in $\mathscr{A}_{m r} \Leftrightarrow A^{\prime} \equiv B^{\prime}\left(\Psi_{P}\right)$ in $\mathscr{A}_{m r}$.

Proof. By Lemma 3, the smallest element in the class containing $A$ modulo ${ }_{P} \Psi$ is $P(A:: P)=P\left(P^{t} A^{\prime}\right)^{\prime}$. The greatest element in the class containing $A$ modulo $\Theta_{P^{t}}$ is $P^{t} A:: P^{t}=\left[P\left(P^{t} A\right)^{\prime}\right]^{\prime}$. Now

$$
A \equiv B\left(\Theta_{P^{t}}\right) \Leftrightarrow\left[P\left(P^{t} A\right)^{\prime}\right]^{\prime}=\left[P\left(P^{t} B\right)^{\prime}\right]^{\prime} \Leftrightarrow P\left(P^{t} A\right)^{\prime}=P\left(P^{t} B\right)^{\prime} \Leftrightarrow A^{\prime} \equiv B^{\prime}\left({ }_{P} \Psi\right) .
$$

The remainder of the lemma is proved similarly.

We say that $P$ in $\mathscr{A}_{n r}$ is left (right) cancellable in $\mathscr{A}_{r m}\left(\mathscr{A}_{m n}\right)$ if $P A=P B(A P=B P)$ implies $A=B$ whenever $A, B \in \mathscr{A}_{r m}\left(A, B \in \mathscr{A}_{m n}\right)$. Note that $P$ is left (right) cancellable if and only if $\Theta_{P}\left({ }_{P} \Theta\right)$ is the identity relation on $\mathscr{A}_{r m}\left(\mathscr{A}_{m n}\right)$. $E$ will denote a matrix with $E_{i j}=\delta_{i j}$.

THEOREM 3. If $P \in \mathscr{C}\left(\mathscr{A}_{n r}\right)$ and $r \leqq m(n \leqq m)$, then the following are equivalent:

(i) $P$ is left (right) cancellable in $\mathscr{A}_{r m}\left(\mathscr{A}_{m n}\right)$.

(ii) There exists $X \in \mathscr{A}_{m n}\left(Y \in \mathscr{A}_{r m}\right)$ such that $X P=E \in \mathscr{A}_{m r}\left(P Y=E \in \mathscr{A}_{n m}\right)$.

(iii) There exists $X \in \mathscr{C}\left(\mathscr{A}_{m n}\right)\left(Y \in \mathscr{C}\left(\mathscr{A}_{r m}\right)\right)$, such that $X P=E \in \mathscr{A}_{m r}\left(P Y=E \in \mathscr{A}_{n m}\right)$.

(iv) $P$ is left (right) cancellable in $\mathscr{C}\left(\mathscr{A}_{r m}\right)\left(\mathscr{C}\left(\mathscr{A}_{m n}\right)\right)$.

Proof. If $P$ in $\mathscr{C}\left(\mathscr{A}_{n r}\right)$ is left cancellable in $\mathscr{A}_{r m}$, then $\Theta_{P}$ is the identity relation on $\mathscr{A}_{r m}$. By Lemma 5, $p \Psi$ is also the identity relation on $\mathscr{A}_{r m}$. The smallest element of the class containing $E$ in $\mathscr{A}_{r m}$ modulo ${ }_{p t} \Psi$ is thus $E=P^{t}\left(E:: P^{t}\right)$. By taking the transpose of each side, we obtain (i) $\Rightarrow$ (ii). Suppose that $X \in \mathscr{A}_{m n}$ and $X P=E$; then $X \leqq E: P$. Now

$$
E=X P \leqq(E: P) P \leqq E .
$$

By Theorem 2, $E: P=\left(E^{\prime} P^{\prime}\right)^{\prime}$ which is in $\mathscr{C}\left(\mathscr{A}_{m n}\right)$. For (iii) $\Rightarrow$ (i), let $X \in \mathscr{C}\left(\mathscr{A}_{m n}\right)$ and $X P=E \in \mathscr{A}_{m r}$. Since two of the three matrices involved are central, $(X, P, A)$ is an associative triple for any $A$ in $\mathscr{A}_{r m}$. Hence $P A=P B$ implies that $E A=E B$, where $E \in \mathscr{A}_{m r}$. If $r \leqq m$, then $E A=E B$ implies that $A=B$. Clearly (i) $\Rightarrow$ (iv). By applying the result (i) $\Rightarrow$ (iii) to matrices over $\mathscr{C}(L)$ we obtain (iv) $\Rightarrow$ (iii).

CoRollary 1. If $P \in \mathscr{C}\left(\mathscr{A}_{n r}\right)$, and if there exists a positive integer $m$ such that $r \leqq m$ $(n \leqq m)$ and $P$ is left (right) cancellable in $\mathscr{A}_{r m}\left(\mathscr{A}_{m n}\right)$, then $P$ is left (right) cancellable in $\mathscr{A}_{r s}$ $\left(\mathscr{A}_{s n}\right)$ for every $r \leqq s(n \leqq s)$.

Proof. Let $A$ be the matrix formed by the first $r$ rows of the matrix described in (iii) of Theorem 3. For any $s \leqq r$, form $A(s)$ by augmenting $A$ to an $s$ rowed matrix whose last $s-r$ rows consist of zeros. Thus $A(s) \in \mathscr{C}\left(\mathscr{A}_{s n}\right)$ and $A(s) P=E \in \mathscr{A}_{s r}$.

Corollary 2. If $P \in \mathscr{C}\left(\mathscr{A}_{n n}\right), n \leqq m$, and $P$ is left (right) cancellable in $\mathscr{A}_{m n}\left(\mathscr{A}_{m n}\right)$, then $P P^{t}=P^{t} P=E$.

Proof. Let $A$ be the matrix formed by the first $n$ rows of the matrix described in (iii) of Theorem 3. Then $A \in \mathscr{C}\left(\mathscr{A}_{n n}\right)$ and $A P=E$. The result now follows from a result of Rutherford [10, §3]. 
3. In this section we consider mappings from $\mathscr{A}_{m n}$ into itself which arise from matrix multiplication. Thus for right (left) multiplication by $P$, we necessarily require that $P \in \mathscr{A}_{n n}$ $\left(P \in \mathscr{A}_{m m}\right)$. After Foulis [5], for an orthomodular lattice $\mathscr{A}$, define $S(\mathscr{A})$ to be the set of all those monotone mappings $\phi: \mathscr{A} \rightarrow \mathscr{A}$ such that there exists at least one, and hence exactly one, monotone mapping $\phi^{*}: \mathscr{A} \rightarrow \mathscr{A}$ with the property that $\left(a^{\prime} \phi\right)^{\prime} \phi^{*} \leqq a$ and $\left(a^{\prime} \phi^{*}\right)^{\prime} \phi \leqq a$ for every $a$ in $\mathscr{A}$. Foulis shows that, if $\phi \in S(\mathscr{A})$, then $\phi$ is residuated, and that $\phi^{*}$ is given by $a \phi^{*}=\left(a^{\prime} \phi^{+}\right)^{\prime}$. Thus $\phi: A \rightarrow A P(\phi: A \rightarrow P A)$ is in $S\left(\mathscr{A}_{m n}\right)$ if and only if $P \in \mathscr{C}\left(\mathscr{A}_{n n}\right)$ $\left(P \in \mathscr{B}\left(\mathscr{A}_{m m}\right)\right)$, and in this case $\phi^{*}$ is given by right (left) multiplication by $P^{t}$. A mapping $\phi$ on a lattice $\mathscr{A}$ is called a closure operator if $a \leqq a \phi$ and $a \phi=(a \phi) \phi$ for all $a$ in $\mathscr{A}$. $\phi$ is called a quantifier on $\mathscr{A}$ if $o \phi=0, a \leqq a \phi$, and $(a \wedge b \phi) \phi=a \phi \wedge b \phi$ for all $a, b$ in $\mathscr{A}$.

Lemma 6. For $P \in \mathscr{A}_{n n}\left(P \in \mathscr{A}_{m m}\right), \phi: A \rightarrow A P(\phi: A \rightarrow P A)$ is a closure operator on $\mathscr{A}_{m n}$ if and only if $E \leqq P, P=P^{2}$, and $(A, P, P)((P, P, A))$ is an associative triple for all $A$ in $\mathscr{A}_{m n}$ *

Proof. If $E \leqq P$, then $A=A E \leqq A P$. Conversely, $E \leqq E \phi=E P=P . \quad A \phi=(A \phi) \phi$ implies that $P=E P=(E P) P=P^{2}$ and $(A P) P=A P=A P^{2}$. If $P=P^{2}$ and $(A, P, P)$ is an associative triple, then $(A P) P=A P^{2}=A P$.

Corollary. If $E \leqq P=P^{2}$ and $P \in \mathscr{C}\left(\mathscr{A}_{n n}\right)\left(P \in \mathscr{C}\left(\mathscr{A}_{m m}\right)\right)$, then $\phi: A \rightarrow A P(\phi: A \rightarrow P A)$ is a closure operator on $\mathscr{A}_{m n}$.

Lemma 7. If $P=P^{t} \in \mathscr{A}_{n n}$, or if $E \leqq P \in \mathscr{A}_{n n}$, then $P=P^{2} \Leftrightarrow P_{i j} \geqq P_{i k} \wedge P_{k j}$ for all $i, j, k=1, \ldots, n$.

Proof. Suppose that $P=P^{t}$ and $P_{i j} \geqq P_{i k} \wedge P_{k j}$. Then $P_{i i} \geqq P_{i k} \wedge P_{k i}=P_{i k}$. Now

$$
P_{i j} \geqq\left(P_{i j} \wedge P_{j j}\right) \vee \bigvee_{k \neq j}\left(P_{i k} \wedge P_{k j}\right)=P_{i j} \vee \bigvee_{k \neq j}\left(P_{i k} \wedge P_{k j}\right) \geqq P_{i j}
$$

i.e. $P_{i j}=P_{i j}^{2}$. Conversely, if $P=P^{t}=P^{2}$, then $P_{i i}=P_{i i} \vee \bigvee_{n \neq i} P_{i k}$, i.e. $P_{i i} \geqq P_{i k}$. Now

$$
P_{i j}=\left(P_{i j} \vee P_{j j}\right) \vee \bigvee_{k \neq j}\left(P_{i k} \wedge P_{k j}\right)=P_{i j} \vee \bigvee_{k \neq j}\left(P_{i k} \wedge P_{k j}\right) \text {. }
$$

Hence $P_{i j} \geqq P_{i k} \wedge P_{k j}$ for all $i, j, k=1, \ldots, n$. If $P \geqq E$, then $P_{i i} \geqq P_{i k}$ and an obvious modification of the above proof establishes the result.

Lemma 8. Given $P \in \mathscr{A}_{n n}\left(P \in \mathscr{A}_{m m}\right)$, the mapping $A \rightarrow A P(A \rightarrow P A)$ is a quantifier on $\mathscr{A}_{m n}$ if and only if $E \leqq P=P^{2}=P^{t}, P \in \mathscr{C}\left(\mathscr{A}_{n n}\right)\left(P \in \mathscr{C}\left(\mathscr{A}_{m m}\right)\right)$, and the columns (rows) of $P$ possess property $\mathscr{D}$ on $L$. (See $[1, \S 1]$ for the definition of property $\mathscr{D}$.)

Proof. For the sufficiency of the conditions, all that remains is to show that

$$
(A \wedge B P) P=A P \wedge B P .
$$

By [1, Lemma 1], $(A \wedge B P) P \leqq A P \wedge(B P) P=A P \wedge B P$. By Lemma $7, P_{h k} \geqq P_{h j} \wedge P_{j k}$, and hence, by property $\mathscr{D}$,

$$
\begin{aligned}
(A P \wedge B P)_{i j} & =V_{k}\left[A_{i k} \wedge P_{k j} \wedge(B P)_{i j}\right]=V_{k}\left[A_{i k} \wedge P_{k j} \wedge V_{h}\left(B_{i h} \wedge P_{h j} \wedge P_{j k}\right)\right] \\
& \leqq V_{k}\left[A_{i k} \wedge P_{k j} \wedge V_{h}\left(B_{i h} \wedge P_{h k}\right)\right]=[(A \wedge B P) P]_{i j}
\end{aligned}
$$


Conversely, if $A \rightarrow A P$ is a quantifier on $\mathscr{A}_{m n}$, then, by Janowitz [7, Theorem 2],

$$
P=P^{2}=P^{t} \in \mathscr{C}\left(\mathscr{A}_{n n}\right) \text {. }
$$

As before, $A \leqq A P$ implies that $E \leqq P$. Let $b \in L$ and let $B$ be such that $B_{i j}=b$ for all $i, j=1, \ldots, n$. Then $A B \wedge B P=(A \wedge B P) P$ becomes $b \wedge V_{k}\left(A_{i k} \wedge P_{k j}\right)=V_{k}\left(A_{i k} \wedge P_{k j} \wedge b\right)$, that is, the columns of $P$ possess property $\mathscr{D}$ on $L$.

Let $\mathscr{A}$ be a lattice with $o$ and 1 , and for $a$ in $\mathscr{A}$ let $\mathscr{A}(o, a)=\{x \in \mathscr{A} \mid x \leqq a\}$. A mapping $\phi: \mathscr{A} \rightarrow \mathscr{A}$ is said to be range closed if $\phi: \mathscr{A} \rightarrow \mathscr{A}(o, 1 \phi)$ is a surjective mapping.

For the next lemma we introduce a notation of Rutherford [10]. If $P$ is a matrix with entries in an orthocomplemented lattice, let $\bar{P}$ be the matrix with $\bar{P}_{i j}=P_{i j} \wedge\left(\bigwedge_{n \neq j} P_{k j}^{\prime}\right)$ and $\underline{P}$ be the matrix with $\underline{P}_{i j}=P_{i j} \wedge\left(\bigwedge_{k \neq i} P_{k j}^{\prime}\right)$.

Lemma 9. Given $P \in \mathscr{C}\left(\mathscr{A}_{n n}\right)\left(P \in \mathscr{C}\left(\mathscr{A}_{m m}\right)\right)$, the mapping $A \rightarrow A P(A \rightarrow P A)$ is range closed in $\mathscr{A}_{m n}$ if and only if any of the following conditions obtain:

(i) $\left(E^{\prime} P^{t}\right)^{\prime} P=E \wedge I P\left(P\left(P^{t} E^{\prime}\right)^{\prime}=E \wedge P I\right)$.

(ii) $\bigvee_{h}\left[P_{h j} \wedge\left(\Lambda_{k \neq j} P_{h k}^{\prime}\right)\right]=\bigvee_{h} P_{h j}$ for all $j=1, \ldots, n,\left(\bigvee_{h}\left[P_{i h} \wedge\left(\Lambda_{k \neq 1} P_{h k}^{\prime}\right)\right]=\bigvee_{h} P_{t h}\right.$ for all $i=1, \ldots, m)$.

(iii) $I \bar{P}=I P(\underline{P I}=P I)$, where $I_{i j}=1$ for all $i, j$.

Proof. First we note that (ii) is the assertion $\left[\left(E^{\prime} P^{t}\right)^{\prime} P\right]_{j j}=[E \wedge I P]_{j j}$ so that (i) $\Rightarrow$ (ii). By [8, Lemma 3.2], $A \rightarrow A P$ is range closed if and only if $\left(A^{\prime} P^{t}\right)^{\prime} P=A \wedge I P$ for all $A$ in $\mathscr{A}_{m n}$. When $A=E$ one obtains the necessity of (i) and (ii). Conversely, $A \geqq\left(A^{\prime} P^{\prime}\right)^{\prime} P$ and $I P \geqq\left(A^{\prime} P^{t}\right)^{\prime} P$ imply that $A \wedge I P \geqq\left(A^{\prime} P^{t}\right)^{\prime} P$ for all $A$ in $\mathscr{A}_{m n}$. Since $\left(A_{i j} \vee P_{h j}^{\prime}\right) \wedge P_{h j}=A_{i j} \wedge P_{h j}$, we find that

$$
\begin{aligned}
{\left[\left(A^{\prime} P^{t}\right)^{\prime} P\right]_{i j} } & =\bigvee_{h}\left[A_{i j} \wedge P_{h j} \wedge \bigwedge_{k \neq j}\left(A_{i k} \vee P_{h k}^{\prime}\right)\right] \\
& \geqq \bigvee_{h}\left[A_{i j} \wedge P_{h j} \wedge \bigwedge_{k \neq j} P_{h k}^{\prime}\right]=A_{i j} \wedge \bigvee_{h}\left[P_{h j} \wedge \bigwedge_{k \neq j} P_{h k}^{\prime}\right] \\
& =A_{i j} \wedge \bigvee_{h} P_{h j}=(A \wedge I P)_{i j}
\end{aligned}
$$

Hence $\left(A^{\prime} P^{t}\right)^{\prime} P=A \wedge I P$ for all $A$ in $\mathscr{A}_{m n}$, and (i) $\Rightarrow$ (ii) $\Rightarrow A \rightarrow A P$ is range closed. (iii) is of course another way of writing (ii).

CoRollary. If $P \in \mathscr{C}\left(\mathscr{A}_{n n}\right)\left(P \in \mathscr{C}\left(\mathscr{A}_{m m}\right)\right)$ and if the elements of each row (column) of $P$ form a mutually orthogonal subset of $L$, that is $P_{i j} \leqq P_{i k}^{\prime}\left(P_{j i} \leqq P_{k i}^{\prime}\right)$ for all $i, j, k$ with $j \neq k$, then the mapping $A \rightarrow A P(A \rightarrow P A)$ is range closed.

Lemma 10. Given $P \in \mathscr{C}\left(\mathscr{A}_{n n}\right)\left(P \in \mathscr{C}\left(\mathscr{A}_{m m}\right)\right) . A \rightarrow A P(A \rightarrow P A)$ is range closed in $\mathscr{A}_{m n}$ if and only if $A^{\prime} P^{t}=B^{\prime} P^{t} \Rightarrow A \wedge I P=B \wedge I P\left(P^{t} A^{\prime}=P^{t} B^{\prime} \Rightarrow A \wedge P I=B \wedge P I\right)$.

Proof. The result follows from [6, Theorem 2].

Corollary 1. If $P \in \mathscr{C}\left(\mathscr{A}_{n n}\right)\left(P \in \mathscr{C}\left(\mathscr{A}_{m m}\right)\right)$ and if $A \rightarrow A P^{t}\left(A \rightarrow P^{t} A\right)$ is range closed, then, for $A \geqq\left(I P^{t}\right)^{\prime}\left(A \geqq\left(P^{t} I\right)^{\prime}\right), A \leftrightarrow A P(A \leftrightarrow P A)$ is a one to one correspondence.

Corollary 2. Suppose that $P \in \mathscr{C}\left(\mathscr{A}_{n n}\right)\left(P \in \mathscr{C}\left(\mathscr{A}_{m m}\right)\right), P$ is row (column) consistent and $A \rightarrow A P^{t}\left(A \rightarrow P^{t} A\right)$ is range closed on $\mathscr{A}_{\mathrm{mn}}$; then $A \leftrightarrow A P(A \leftrightarrow P A)$ is a one to one correspondence on $\mathscr{A}_{m n}$. 
Let $\mathscr{A}$ be an orthomodular lattice and let $e \in \mathscr{A}$. Define a mapping $\phi_{e}$ by $a \phi_{c}=\left(a \vee e^{\prime}\right) \wedge e$ for $a$ in $\mathscr{A}$. Such mappings are called Sasaki projections and are especially interesting members of $S(\mathscr{A})$. Foulis notes that when $\phi=\phi^{2}=\phi^{*} \in S(\mathscr{A}), \phi$ is a Sasaki projection if and only if $\phi$ is range closed. Thus we have the following:

TheOREM 4. Let $P \in \mathscr{C}\left(\mathscr{A}_{n n}\right)\left(P \in \mathscr{C}\left(\mathscr{A}_{m m}\right)\right)$, and let $P=P^{2}=P^{t}$. The mapping $A \rightarrow A P$ $(A \rightarrow P A)$ is a Sasaki projection in $\mathscr{A}_{m n}$ if and only if $P$ is a diagonal matrix, i.e. $P_{i j}=o$ for $i \neq j$.

Proof. If $P$ is a diagonal matrix, then, by the Corollary to Lemma 9, the mapping $A \rightarrow A P$ is range closed and hence is a Sasaki projection. Conversely, by Lemma $7, P_{i j} \geqq P_{i k} \wedge P_{k j}$ and $P_{j j} \geqq P_{j k}$. Since $P_{h j} \wedge P_{h h}=o$, it follows from Lemma 9 that

$$
P_{j k} \leqq P_{j j}=\bigvee_{h} P_{h j}=\bigvee_{h}\left[P_{h j} \wedge \bigwedge_{k \neq j} P_{h k}^{\prime}\right]=P_{j j} \wedge \bigvee_{k \neq j} P_{j k}^{\prime} \leqq P_{j k}^{\prime} \text { for } j \neq k
$$

Thus $P_{j k}=P_{j k} \wedge P_{j k}^{\prime}=o$ for $j \neq k$.

\section{REFERENCES}

1. J. H. Bevis, Matrices over orthomodular lattices, Glasgow Math. J. 10 (1968), 55-59.

2. G. Birkhoff, Lattice theory, Amer. Math. Soc. Colloquium Publications, Vol. 25, rev. ed. (New York, 1948). $185-190$.

3. T. S. Blyth, Residuation theory and Boolean matrices, Proc. Glasgow Math. Assoc. 6 (1964),

4. R. Croisot, Applications residuées, Ann. Sci. Ecole Norm. Sup. (3) 73 (1956), 453-474.

5. D. J. Foulis, Baer *-semigroups, Proc. Amer. Math. Soc. 11 (1960), 648-654.

6. D. J. Foulis, Conditions for the modularity of an orthomodular lattice, Pacific J. Marh. 11 (1961), 889-895.

7. M. F. Janowitz, Quantifiers and orthomodular lattices, Pacific J. Math. 13 (1963), 1241-1249.

8. M. F. Janowitz, A semigroup approach to lattices, Canad. J. Math. 18 (1966), 1212-1223.

9. I. Molinaro, Demi-groupes résidutifs, J. Math. Pures Appl. 39 (1960), 319-356.

10. D. E. Rutherford, Inverses of Boolean matrices, Proc. Glasgow Math. Assoc. 6 (1963), 49- 53

Virginia Polytechnic Institute

BLACKSBURG, VIRGINIA 